\title{
Efeito do treinamento vibratório na força muscular e em testes funcionais em idosos fisicamente ativos
}

\author{
Effects of a whole body vibration training program on strength, \\ power, and functional tests in the physically active elderly
}

\author{
Renata Gonçalves da Silva ${ }^{1,2}$ \\ Rosana Andreotti \\ Paula Regina Gehring ${ }^{2}$ \\ Marcelo Eduardo de Souza Nunes 2 \\ Lilian Wallerstein ${ }^{2}$ \\ Maria Cecília Oliveira da Fonseca ${ }^{2}$ \\ Suely Santos ${ }^{3}$ \\ Valmor Tricoli 4 \\ Carlos Ugrinowitsch ${ }^{4}$
}

1 Universidade Paulista.

2 Universidade de São Paulo. Escola de Educação Física e Esporte. São Paulo, SP.

3 Universidade de São Paulo. Departamento de Pedagogia do Movimento Humano. Escola de Educação Física e Esporte. São Paulo, SP.

4 Universidade de São Paulo. Departamento de Esporte. Escola de Educação Física e Esporte. São Paulo, SP.

Recebido em 18/12/07 Aprovado em 24/07/08
Resumo - O objetivo do presente estudo foi investigar o efeito do treinamento de vibração na força e na potência musculares, a $60^{\circ} . \mathrm{s}^{-1}$ e a $180^{\circ} . \mathrm{s}^{-1} \mathrm{e}$ na funcionalidade de idosos. Participaram do estudo quarenta e sete idosos que já frequentavam um programa de exercícios físicos. Os sujeitos foram divididos em dois grupos: grupo experimental (GE) ( $n=24$, $70.7 \pm 5.8$ anos, $62.7 \pm 12.3 \mathrm{~kg}$ e $155.9 \pm 8.0 \mathrm{~cm})$ e grupo controle $(\mathrm{GC})(\mathrm{n}=23,70.0 \pm 5.7$ anos, $65.3 \pm 10.7 \mathrm{~kg}$ e $157.9 \pm 6.6 \mathrm{~cm}$ ). Adicionalmente ao programa de exercícios físicos, o GE realizou o treinamento de vibração durante 13 semanas, sendo 2 vezes por semana, enquanto que o GC continuou apenas com o programa regular de exercícios físicos. $\mathrm{O}$ treinamento de vibração não foi efetivo em melhorar a força, a potência e o desempenho nos testes funcionais dos idosos. No entanto, encontra-se efeito de tempo para os testes de força dos flexores de cotovelo, à distância percorrida no teste de caminhada de 6 minutos e a redução no tempo para realizar o percurso no teste de agilidade/equilíbrio. Os resultados sugerem que o treinamento de vibração parece não ser eficiente para modificar a funcionalidade bem como a força e a potência musculares em idosos fisicamente ativos.

Palavras-chave: Envelhecimento; Funcionalidade; Exercício físico

Abstract - The aim of this study was to investigate the effect of a whole body vibration training program on knee extensor isokinetic peak torque, average power, and functional tests in the physically active elderly. Forty-seven subjects, who participated in a training program for the elderly, were recruited. The subjects were divided into two groups: whole body vibration group (WBV) $(n=24,70.7 \pm 5.8$ years, $62.7 \pm 12.3 \mathrm{~kg}$ and $155.9 \pm 8.0 \mathrm{~cm})$ and control group $(n=23.70 .0 \pm 5.7$ years, $65.3 \pm 10.7 \mathrm{~kg}$ and $157.9 \pm 6.6 \mathrm{~cm}$ ). The WBS group underwent whole body vibration exercise and their regular training program for 13 weeks, 2 times per week, whereas the control group performed the regular training program only. Whole body vibration training was not effective in increasing isokinetic peak torque, average power, or performance in the functional tests. On the other hand, the regular training program was effective in increasing elbow flexor strength endurance, distance covered in the 6-min walk test, and speed in the timed up and go test. The results suggest that vibration training is not effective in modifying functionality or muscle strength and power in the physically active elderly.

Key words: Aging; Functionality; Physical exercise. 


\section{INTRODUÇÃO}

O Brasil vem passando por um processo de envelhecimento nas últimas décadas. $\mathrm{Na}$ década de 40 do século passado, as pessoas com 60 anos ou mais representam $4,1 \%$ da população brasileira, enquanto que em 2000 elas já representavam 8,6\%1. Um estudo realizado no início dos anos 902, com 1602 idosos (60 anos ou mais), em dez subdistritos do município de São Paulo, revelou que 53\% desses idosos eram totalmente independentes para suas atividades rotineiras e instrumentais de vida diária como preparar refeições, fazer a limpeza da casa, tomar remédios, pentear cabelos, andar no plano, comer, tomar banho, vestir-se, deitar/levantar da cama, ir ao banheiro em tempo, cortar as unhas dos pés, subir um lance de escada, ir a um lugar necessitando de condução e ir andando a um lugar perto de casa. Enquanto 29\% dos idosos necessitavam de ajuda parcial ou total para realizar até três dessas atividades, $10 \%$ precisavam de ajuda em mais de três e menos de sete e $8 \%$ necessitavam de ajuda parcial ou total para realizar pelo menos sete dessas atividades.

Essa redução na capacidade do indivíduo em levar uma vida independente acontece, também, em função do declínio da potência e da força muscular, que ocorre com o avançar da idade ${ }^{3,4}$. O $\mathrm{ACSM}^{5}$ enfatiza que o declínio da força muscular relacionado ao envelhecimento produz consequências significativas sobre a capacidade funcional. Mesmo em atividades básicas da vida diária, como caminhar, subir escadas e sentar em uma cadeira, os idosos passam a apresentar limitações que não os permitem realizá-las autonomamente ${ }^{3,4,6}$. A literatura específica também indica que esse declínio é a principal causa de quedas e fraturas de quadril ${ }^{6}$.

O treinamento físico pode retardar o declínio, ou aumentar tanto a força quanto a potência muscular e, consequentemente, a capacidade funcional do indivíduo ao longo do tempo ${ }^{7,8,9}$. Uma das formas de treinamento que vem sendo utilizada para manter a funcionalidade de idosos é o método chamado Whole Body Vibration (WBV). Nesse método, o sujeito fica sobre uma plataforma que gera uma vibração sinosoidal em diversas freqüências e amplitudes, que é transmitida para o corpo estimulando os fusos musculares. A ativação dos fusos musculares produz um reflexo vibratório tônico que ativa os motoneurônios alfa. Consequentemente, uma maior população de motoneurônios ativa é responsável por uma maior produção de força e potência.

Alguns estudos têm indicado que a maior produção de força e potência, após o treinamento vibratório (TV), melhora a performance em testes funcionais e modifica o padrão da marcha de idosos (Russo, 2003; Iwamoto, 2004; Verschuren, 2004). Contudo, não há dados na literatura que indiquem que o TV pode produzir melhoras adicionais na força, na potência e na performance de testes funcionais de idosos já fisicamente ativos. Sendo assim, o presente estudo teve por objetivo, verificar o efeito de três meses de treinamento na plataforma vibratória, no desempenho de força e potência muscular e em um teste utilizado para avaliar o desempenho em testes funcionais em idosos que já realizam um programa de exercícios físicos.

\section{PROCEDIMENTOS METODOLÓGICOS}

\section{Amostra}

A amostra foi composta por 47 idosos fisicamente ativos. Os indivíduos faziam parte de um programa de condicionamento físico (CEFI - Curso de Educação Física para Idosos) na Escola de Educação Física e Esporte da Universidade de São Paulo (EEFE-USP). Os idosos frequentavam esse programa duas vezes por semana. Todos os participantes tinham autorização médica para participar dos testes e do programa. Foi solicitado aos indivíduos que mantivessem suas atividades diárias durante toda a duração do estudo. Todos foram informados dos possíveis riscos, desconfortos e benefícios do estudo e foi obtido o consentimento por escrito de todos os participantes. .

\section{Formação dos Grupos}

Após o processo de seleção, os sujeitos foram divididos em dois grupos (experimental e controle). O grupo experimental foi composto por 5 homens e 19 mulheres $(70,7 \pm 5,8$ anos, $62,7 \pm 12,3$ quilos e $155,9 \pm 8,0$ centímetros). O grupo controle tinha 7 homens e 16 mulheres $(70,0 \pm 5,7$ anos, $65,3 \pm 10,7$ quilos e $157,9 \pm 6,6$ centímetros). A alocação dos sujeitos aos grupos não foi feita de forma aleatória para permitir que os grupos fossem equalizados quanto ao nível de aptidão física pré-treinamento. Primeiro, os indivíduos realizaram os pré-testes e foram divididos em quartis. Após isso, os indivíduos dentro dos quartis foram alocados aleatoriamente nos grupos experimental (GE) e controle (GC). Dessa forma, os grupos tinham o mesmo número de indivíduos de cada quartil e, por isso, condição inicial similar.

\section{Grupo Controle}

Os sujeitos participaram somente do programa do CEFI (Curso de Educação Física para Idosos) duas 
Tabela 1. Exercícios, freqüência, número de séries, duração, e sessões do programa de treinamento na plataforma vibratória para idosos fisicamente ativos.

\begin{tabular}{|c|c|c|c|c|}
\hline Exercício & $\begin{array}{l}\text { Freqüência } \\
(\mathrm{Hz})\end{array}$ & $\mathrm{n}^{\circ}$ séries & $\begin{array}{c}\text { Duração } \\
\text { (s) }\end{array}$ & Sessões \\
\hline Agachamento & 30 & 2 & 30 & $1^{\mathrm{a}}$ a $4^{\underline{a}}$ \\
\hline Agachamento & 35 & 3 & 45 & $9^{\underline{a}}$ a $10^{\underline{a}}$ \\
\hline Agachamento & 35 & 3 & 45 & $11^{\mathrm{a}}$ a $12^{\underline{a}}$ \\
\hline Agachamento (pés voltados para fora) & 35 & 2 & 30 & $11^{\mathrm{a}}$ a $12^{\mathrm{a}}$ \\
\hline Agachamento & 35 & 3 & 45 & $13^{\mathrm{a}}$ a $16^{\underline{a}}$ \\
\hline Agachamento (pés voltados para fora) & 35 & 2 & 30 & $13^{\underline{a}}$ a $16^{\underline{a}}$ \\
\hline Agachamento (pés voltados para fora) & 40 & 2 & 45 & $17^{\underline{a}}$ a $20^{\underline{a}}$ \\
\hline Agachamento unilateral & 40 & 1 & 30 & $17^{\mathrm{a}}$ a $20^{\mathrm{a}}$ \\
\hline Agachamento & 40 & 3 & 45 & $21^{\mathrm{a}}$ a $23^{\mathrm{a}}$ \\
\hline Agachamento (pés voltados para fora) & 40 & 2 & 45 & $21^{\mathrm{a}}$ a $23^{\mathrm{a}}$ \\
\hline Agachamento unilateral & 40 & 1 & 30 & $21^{\mathrm{a}}$ a $23^{\underline{a}}$ \\
\hline Agachamento & 40 & 3 & 60 & $24^{\mathrm{a}}$ a $26^{\mathrm{a}}$ \\
\hline Agachamento (pés voltados para fora) & 40 & 2 & 45 & $24^{\underline{a}}$ a $26^{\underline{a}}$ \\
\hline
\end{tabular}

vezes por semana na EEFE-USP. Em linhas gerais, o treinamento tinha duração de 1 hora, com exercícios de propriocepção/equilíbrio (10 min.), aeróbio (20 min.), força (20 min.) e flexibilidade (10 min.).

\section{Grupo Experimental}

Além de participarem do programa do CEFI como o grupo controle, os sujeitos realizaram 26 sessões de um treinamento com exercícios isométricos na plataforma de vibração Power Plate ${ }^{\circledR}$. Os exercícios foram: agachamentos com os pés paralelos, joelhos flexionados entre 110 e $130^{\circ}$ (angulação mais confortável) e flexão do quadril de aproximadamente $140^{\circ}$ (angulação mais confortável); agachamento com os pés voltados para fora e agachamento unilateral com os mesmos graus de flexão de joelho e quadril. A progressão do treinamento foi feita através do aumento do número de séries, da amplitude ou da intensidade da vibração.

Nas oito primeiras sessões foram feitas séries de agachamento com os pés paralelos. Da nona até a $12^{a}$ sessão foram incluídas séries de agachamento com os pés voltados para fora, e da $13^{a}$ até o final do estudo incluiu-se o agachamento unilateral. $\mathrm{O}$ número de séries e a duração das séries estão descritos na Tabela 1 . Até a $16^{\mathrm{a}}$ sessão a amplitude foi baixa (2-4mm) e da $17^{\mathrm{a}}$ até a $26^{\mathrm{a}}$ a amplitude foi alta (4$6 \mathrm{~mm})$. Utilizou-se uma freqüência de $30 \mathrm{~Hz}$ nas oito primeiras sessões, de $35 \mathrm{~Hz}$ da nona sessão até a $16^{\mathrm{a}}$ sessão e de $40 \mathrm{~Hz}$ a partir da $17^{\mathrm{a}}$ sessão até o final do estudo. As sessões tiveram duração máxima de 20 minutos, incluindo aquecimento e alongamento.

\section{Avaliações}

As avaliações foram realizadas em duas sessões, tanto no pré quanto no pós-teste. Na primeira sessão, foram realizadas medidas antropométricas e uma bateria de testes funcionais para avaliar as atividades da vida diária - desenvolvida para testar força muscular de membros inferiores e superiores, flexibilidade de membros superiores e inferiores, agilidade, equilíbrio dinâmico e resistência aeróbia. Já na segunda sessão foi avaliado o pico de torque e a potência média obtida em um dinamômetro isocinético.

- Medidas Antropométricas (estatura, massa corporal e índice de massa corporal):

A medida da estatura foi realizada em um estadiômetro com precisão de $0,1 \mathrm{~cm}$ e a de massa corporal total, em uma balança eletrônica da marca Filizola ${ }^{\circledR}$ com $0,1 \mathrm{~kg}$ de precisão. $\mathrm{O}$ índice de massa corpo- 
ral (IMC) foi calculado através da fórmula massa corporal $\cdot$ estatura $^{-2}\left(\mathrm{~kg} \cdot \mathrm{m}^{-2}\right)$.

\section{Bateria de Testes Funcionais ${ }^{10,11}$}

- Flexibilidade (Sentar na Cadeira e Alcançar - SCA):

O sujeito sentava na borda da cadeira, com uma perna estendida o máximo possível e tornozelo em posição neutra $\left(90^{\circ}\right)$ e descia lentamente o tronco com os braços estendidos e as mãos sobrepostas. A outra perna tinha o joelho flexionado à aproximadamente $90^{\circ}$. A extremidade do hálux correspondia ao ponto zero. Não alcançando esse ponto, o resultado era negativo (distância que faltava até o hálux) e, ultrapassando-o, o resultado era positivo (distância alcançada depois do hálux). Foram realizadas duas tentativas, e o melhor escore foi utilizado.

- Agilidade/Equilíbrio dinâmico (Levantar-ir-eVoltar - LIV):

O sujeito iniciava o teste sentado em uma cadeira, mãos nas coxas e pés apoiados no solo. Ao sinal do avaliador, o sujeito deveria levantar e caminhar o mais rápido possível, sem correr, contornando um cone a uma distância de 2,44 m e retornar à posição inicial. $\mathrm{O}$ cronômetro era acionado a partir do sinal do avaliador e, novamente, quando o sujeito se sentava totalmente na cadeira. Após demonstração, o sujeito fazia uma tentativa do teste para familiarizarse e logo após, eram realizadas duas tentativas. Utilizamos o melhor escore (tempo em segundos).

- Flexibilidade (Mãos nas Costas - MC):

Em pé, o sujeito colocava sua mão de preferência nas costas, passando o braço por sobre o ombro. A palma da mão deveria estar voltada para as costas com os dedos estendidos, tentando alcançar a maior distância (em direção aos quadris). A outra mão também deveria ser colocada nas costas, porém com o braço passando pela lateral do corpo. O objetivo do teste foi aproximar (ou sobrepor) as mãos o máximo possível. Após demonstração do avaliador, o sujeito teve duas tentativas e, como resultado, utilizamos o melhor escore. A medida da distância entre os dedos médios era feita $\mathrm{em} \mathrm{cm}$. Um escore negativo foi dado quando os dedos não conseguiam se tocar e positivo quando os dedos se sobrepunham.

- Força de membros inferiores (Sentar e Levantar - SL):

O teste começava com o sujeito sentado com as costas encostadas na cadeira e os pés apoiados no chão.
Ao sinal do avaliador, o sujeito deveria levantar e voltar à posição inicial. $\mathrm{O}$ sujeito foi encorajado a completar o maior número de repetições possível no período de 30 segundos. Antes de iniciar o teste, o avaliador demonstrava o exercício e então o sujeito praticava de uma a três repetições para familiarização à tarefa, iniciando o teste em seguida.

- Força de membros superiores (Flexão do Cotovelo - FC):

O sujeito começava sentado com as costas apoiada na cadeira, braços estendidos e executava o movimento de flexão e extensão do cotovelo do membro dominante (Carga: 5 libras para mulheres e 8 libras para homens). $O$ teste começava ao sinal do avaliador, cabendo ao sujeito flexionar e estender o cotovelo. O sujeito foi encorajado a completar o maior número de repetições possível no período de 30 segundos. Após demonstração do teste, o sujeito praticava de uma a três repetições para familiarizarse à tarefa, iniciando o teste em seguida.

- Resistência Aeróbia (Caminhada 6min - C6M): Distância percorrida, caminhando o mais rápido possível, em um período de 6 minutos. O percurso retangular tinha uma distância total de $45,72 \mathrm{~m}$ e foi demarcado por cones a cada $4,57 \mathrm{~m}$. Os sujeitos foram avisados quando faltavam 2 minutos e $1 \mathrm{mi}$ nuto para o término do tempo. Ao final do tempo, os sujeitos paravam onde estavam e então, era feita a medida da distância percorrida.

- Força e potência muscular:

Foram realizadas avaliações no dinamômetro Isocinético, modelo Rev 9000 da Technogym ${ }^{\circledR}$. O teste foi composto de duas séries, sendo uma série de cinco repetições a $60 \%$ e uma série de cinco repetições a $180 \%$ (com objetivo de mensurar força e potência), com intervalo de 90 segundos entre as séries. Os sujeitos foram encorajados a realizar o máximo de força de extensão de joelho do membro dominante. A força máxima e o trabalho realizado foram medidos a cada movimento e foram utilizados os melhores resultados obtidos em cada série para análise. Antes das avaliações propriamente ditas, os sujeitos realizaram duas séries de cinco repetições em nível submáximo com as mesmas velocidades dos testes para se familiarizarem ao tipo de resistência oferecida pelo dinamômetro. Contudo, antes da familiarização e dos testes no aparelho isocinético, foi realizado um período de aquecimento no ciclo ergômetro com carga de 25 watts, com duração de cinco minutos e uma sequ- 
Tabela 2. Resultados de IMC ( $\left.\mathrm{kg} / \mathrm{m}^{-2}\right)$, sentar e levantar (SL) (repetições), flexão de cotovelo (FC) (repetições), sentar na cadeira e levantar (SCA) (repetições), mãos nas costas (MC) (distância - cm), agilidade (segundos), caminhada 6 minutos (C6m) (distância - metros) nos grupos de treinamento vibratório (TV) e grupo controle (GC) pré- e pós treinamento na plataforma vibratória em idosos fisicamente ativos (média \pm desvio padrão).

\begin{tabular}{lcccc}
\hline & \multicolumn{2}{c}{ Grupo Experimental } & \multicolumn{2}{c}{ Grupo Controle } \\
& Pré-teste & Pós-teste & Pré-teste & Pós-teste \\
\hline IMC & $25,7 \pm 4,2$ & $25,3 \pm 3,7$ & $26,2 \pm 3,6$ & $26,6 \pm 3,8$ \\
SL & $20,0 \pm 3,7$ & $19,8 \pm 3,0$ & $17,5 \pm 3,8$ & $16,9 \pm 4,9$ \\
FC* & $21,7 \pm 3,7$ & $25,2 \pm 3,4$ & $19,6 \pm 4,3$ & $21,9 \pm 4,9$ \\
SCA & $3,3 \pm 9,8$ & $5,6 \pm 13,6$ & $4,9 \pm 11,1$ & $3,6 \pm 13,9$ \\
MC & $-4,1 \pm 9,2$ & $-4,3 \pm 9,2$ & $-3,6 \pm 10,3$ & $-5,4 \pm 11,2$ \\
Agilidade* & $5,2 \pm 0,6$ & $4,8 \pm 0,5$ & $5,5 \pm 1,2$ & $5,3 \pm 1,2$ \\
C6M* $^{*}$ & $526,2 \pm 81,6$ & $603,0 \pm 63,6$ & $515,9 \pm 96,7$ & $568,2 \pm 101,7$ \\
\hline
\end{tabular}

*Efeito principal de tempo $(\mathrm{p}<0,05)$

Tabela 3. Resultados de Pico de Torque $60^{\circ} \bullet \mathrm{s}^{-1}$ (Watt), Potência $60^{\circ} \bullet \mathrm{s}^{-1}$ (Watt), Pico de Torque $180^{\circ} \bullet \mathrm{s}^{-1}(\mathrm{Watt})$, Potência $180^{\circ} \bullet \mathrm{s}^{-1}$ (Watt) nos grupos de treinamento vibratório (TV) e grupo controle (GC) pré- e pós treinamento na plataforma vibratória em idosos fisicamente ativos (média \pm desvio padrão).

\begin{tabular}{lcccc}
\hline & \multicolumn{2}{c}{ Grupo Experimental } & \multicolumn{2}{c}{ Grupo Controle } \\
& Pré-teste & Pós-teste & Pré-teste & Pós-teste \\
\hline Pico Torque $60^{\circ} \bullet \mathrm{s}^{-1}$ & $85,3 \pm 34,5$ & $86,3 \pm 39,7$ & $88,0 \pm 37,6$ & $99,0 \pm 20,0$ \\
Potência $60^{\circ} \bullet \mathrm{s}^{-1}$ & $52,0 \pm 41,4$ & $57,2 \pm 32,9$ & $55,3 \pm 30,5$ & $58,7 \pm 11,4$ \\
Pico Torque $180^{\circ} \bullet \mathrm{s}^{-1}$ & $64,6 \pm 26,5$ & $66,0 \pm 32,1$ & $67,3 \pm 22,1$ & $69,0 \pm 10,1$ \\
Potência $180^{\circ} \bullet \mathrm{s}^{-1}$ & $69,2 \pm 37,0$ & $88,2 \pm 60,4$ & $104,6 \pm 99,4$ & $86 \pm 31,0$ \\
\hline
\end{tabular}

ência de exercícios de flexibilidade de quadríceps por mais cinco minutos.

\section{Análise Estatística}

Para cada variável dependente, foi usado um modelo misto, tendo o tratamento (treinamento na plataforma vibratória e controle) e o momento (pré e pós) como fatores fixos e sujeitos como fator aleatório. No caso de valores de F significantes, comparações múltiplas foram realizadas com ajustamento de Tukey. O nível de significância foi fixado em $\mathrm{p}<0,05$.

\section{RESULTADOS}

O treinamento na plataforma vibratória não foi efetivo em melhorar o desempenho nos testes funcionais em idosos. Contudo, o programa de condicionamento físico ao que os sujeitos de ambos os grupos participavam foi eficiente em aumentar a força dos flexores de cotovelo, a distância percorrida no teste de caminhada de 6 minutos e em diminuir o tempo para cumprir o percurso no teste de agilidade ( $\mathrm{p}<0,05$, Tabela 2$)$.

O modelo de treinamento vibratório utilizado não foi eficiente em aumentar tanto o torque quanto a potência dos músculos extensores do joelho. Além disso, o programa de exercício físico utilizado também não produziu melhoras nas variáveis descritas abaixo (Tabela 3).

\section{DISCUSSÃO}

O programa de condicionamento físico foi eficiente em aumentar a resistência de força dos flexores do cotovelo, a velocidade no teste de agilidade e a distância percorrida no teste de caminhada de 6 minutos em indivíduos fisicamente ativos. Tanto o treinamento na plataforma vibratória quanto o programa de exercícios físicos não foram eficientes em aumentar o torque e a potência dos músculos extensores do joelho.

O teste de agilidade (LIV) envolve potência, velocidade, agilidade e equilíbrio dinâmico ${ }^{11}$, capacidades motoras que declinam com o envelhecimento, aumentam o risco não só de quedas e fraturas, como também diminuem o nível de independência do idoso. Era esperado que o TV melhorasse o desempenho dos idosos nesse teste pelo aumento da força motora dos mesmos. No entanto, os resultados diferem dos achados de outros pesquisadores ${ }^{12-14}$.

Os estudos de Bautmans et al. ${ }^{12}$ e Bruyere et al..$^{13}$ utilizaram um modelo de TV com características semelhantes ao do presente estudo $(26-50 \mathrm{~Hz}, 2-7 \mathrm{~mm}$, 240 a $400 \mathrm{~s}$ de exposição à vibração por sessão e 
duração de seis semanas - 18 sessões). Eles reportaram melhoras de $21,6 \%$ e $30,5 \%$, respectivamente, no desempenho no teste de LIV, após o treinamento vibratório. Um ponto importante é que ambos os estudos encontraram melhora também no teste de Tinetti (equilíbrio) e atribuíram o aumento de desempenho no LIV à melhora no equilíbrio, já que não encontraram aumentos significantes de força e potência muscular, os quais também poderiam justificar a melhora no desempenho. A ausência de ganho na força e na potência muscular após o TV, no presente estudo, indicada pela manutenção nos valores de pico de torque e de potência média no teste isocinético, pode ser então a razão pela qual não foram observadas mudanças no teste LIV, já que não foi realizada uma medida precisa de equilíbrio. Contudo, os autores não descreveram o nível de condicionamento físico inicial dos sujeitos. Dessa forma, apesar da diferença na distância percorrida no LIV (2,44m contra 3,0m), seus resultados (15,3s e 36,1s), comparados com o resultado do presente estudo (5,2s), sugerem que os sujeitos dos estudos Bautmans et al. ${ }^{12} \mathrm{e}$ Bruyere et al..$^{13}$ eram muito debilitados ${ }^{16,17}$, o que pode ser um fator relevante para a melhora encontrada no desempenho.

Outros estudos avaliaram o efeito do TV no equilíbrio, utilizando outros métodos de avaliação (variações de testes em plataformas de equilíbrio) e também encontraram melhora no desempenho com o TV ${ }^{15,18}$. No entanto, esses estudos não realizaram o teste de LIV, o que torna difícil a comparação.

Não houve aumento significante na flexibilidade, tanto para membros superiores quanto para inferiores, em nenhum dos grupos. Esses resultados são semelhantes aos encontrados por Bautmans et al..$^{12}$, que utilizaram os mesmos protocolos de avaliação. Contudo, o estudo de Fagnani et al. ${ }^{19}$ reportou melhora na flexibilidade (13\%), medida com o teste de sentar e alcançar, em mulheres atletas (de 21 a 27 anos), após oito semanas de TV. As sobrecargas eram semelhantes (freqüência $35 \mathrm{~Hz}$, amplitude $4 \mathrm{~mm}$ ), com o uso do peso corporal. $\mathrm{O}$ treinamento começou com 3 séries de 20s, chegando a 4 séries de 60s para o agachamento, e 4 séries de 30s para o agachamento unilateral, em um total de oito semanas, com três sessões de treino por semana. Da mesma forma, o estudo de Issurin ${ }^{18}$ reportou um aumento de 43,6\% no mesmo teste com a utilização de vibração direta em atletas que treinaram três vezes por semana, durante três semanas. Issurin et al. ${ }^{20}$ e Fagnani et al. ${ }^{19}$ sugerem que essa melhora pode acontecer em função do aumento do limiar de dor, bem conhecido como barreira natural para a flexibilidade e aumento da temperatura e do fluxo sanguíneo. Outro mecanismo é baseado na excitação do órgão tendinoso de golgi (OTG), que inibi a contração muscular, seguido pelo seu relaxamento. Talvez essa melhora na flexibilidade em atletas possa ser atribuída ao treinamento específico de suas modalidades esportivas e não ao TV. Atletas apresentam uma treinabilidade menor do que a de idosos e por isso não deveriam melhorar a flexibilidade, enquanto que se o TV produzisse melhorias nessa capacidade os idosos deveriam apresentar. Outro fator pode ser o efeito da vibração direta (aplicada diretamente no grupo muscular) já que ela parece ser mais eficiente em estimular os mecanismos citados acima ${ }^{20}$.

O que difere da grande maioria dos estudos com idosos ${ }^{8,21,22}$ e também com jovens ${ }^{23,24}$ são as variáveis força e potência muscular, que não melhoraram após o período de treinamento.

O número de sessões, intensidade, tempo de exposição à vibração e exercícios realizados na plataforma são fatores importantes a serem considerados nessa análise. Os exercícios utilizados nos estudos eram semelhantes (variações de agachamento). Com isso, as diferenças entre os estudos parecem estar relacionadas às características do protocolo de TV ou às características dos sujeitos.

Os estudos que encontraram aumento na força dinâmica utilizaram uma freqüência de vibração semelhante à nossa $(35-40 \mathrm{~Hz})$, porém diferiam na amplitude da vibração e no número de sessões de treinamento. Em relação ao primeiro fator, no estudo de Verschueren et al..$^{22}$ foi utilizada uma amplitude de 1,7-2,5 mm e no de Roelants et al. ${ }^{8}$ de $2-5,5 \mathrm{~mm}$, obtendo ganhos de $15 \%$ na força isométrica e 16,5\% na força dinâmica dos extensores do joelho e de $12,4 \%$ e $12,1 \%$ para as mesmas variáveis, respectivamente. Dessa forma, a amplitude do treino vibratório não parece ter efeito sobre a magnitude das adaptações. Em relação ao segundo fator, Verschueren et al. ${ }^{22}$ et al. e Roelants et al. ${ }^{8}$ utilizaram 72 sessões de treinamento de vibração, porém os percentuais de aumento reportados no último estudo foram obtidos com apenas 36 sessões de TV. Esses dados indicam que o número de sessões também não parece ser um fator determinante na magnitude das adaptações encontradas. $\mathrm{O}$ único fator, relacionado às características do treinamento, que poderia explicar então as diferenças em ganhos de força entre o nosso estudo e os citados é a característica da amostra, já que eles utilizaram idosos sedentários enquanto nós utilizados idosos fisicamente ativos. 
Roelants et $\mathrm{al} .{ }^{8}$ encontraram, também, melhora no desempenho do salto vertical com contra-movimento para o grupo que praticou o TV $(19,4 \%)$. Russo et al. ${ }^{21}$ encontraram melhora no salto vertical (5\%) para o TV após 24 semanas de treinamento (2x semana, iniciando com 3x 1 min e no final do estudo 3x 2 min; a freqüência de 12 para $28 \mathrm{~Hz}$ ). No presente estudo, não foi encontrado aumento na potência média nos testes isocinéticos. A diferente natureza dos testes (altura do salto vertical e potência média no dinamômetro isocinético) pode ter induzido a ausência de ganhos de potência no nosso estudo devido à baixa sensibilidade do teste isocinético.

O aumento da resistência de força dos flexores do cotovelo que apresentou efeito de tempo demonstra que apenas o programa de exercício físico foi eficiente para a melhora dessas variáveis. Isso pode ter ocorrido em função da utilização de exercícios de força para membros superiores, no programa de condicionamento físico, já que esse grupo muscular não foi treinado no nosso programa de TV.

Houve um efeito de tempo na distância percorrida no teste de 6 minutos, o que mostra que o programa de exercício físico foi o responsável por esse aumento. Esse aumento é importante para melhora da qualidade de vida do idoso, considerando que esse teste vem sendo relacionado com desempenho funcional e que os idosos necessitam de um adequado nível de desempenho aeróbio para algumas atividades diárias, como caminhada, deslocamento dentro de casa, passeio ao shopping e até mesmo o ato de ir ao banheiro ${ }^{11,25}$. Como o teste está relacionado ao desempenho aeróbio, o treinamento específico, aeróbio, realizado nas sessões de treinamento pelos dois grupos parece justificar essa melhora.

\section{CONCLUSÃO}

Concluindo, os resultados sugerem que o TV, realizado durante 13 semanas (26 sessões) em idosos fisicamente ativos, parece não ser eficiente para aumentar a força muscular e o desempenho em testes funcionais. Dessa forma, coloca-se em dúvida a eficiência desse método de treinamento no desenvolvimento da força motora, mesmo em indivíduos idosos.

\section{REFERÊNCIAS BIBLIOGRÁFICAS}

1. Instituto Brasileiro de Geografia e Pesquisa. Tendências demográficas: uma análise da população com base nos re- sultados dos censos demográficos de 1940 e 2000. Disponível em: <http://www.ibge.gov.br/home/presidencia/noticias/ noticia_impressao.php?id_noticia $=892>$. [24 abr 2008].

2. Ramos RL, Rosa TEC, Oliveira ZM, Medina MCG, Santos, FRG. Profile of the elderly in an metropolitan area of Southeastern Brazil: results of a household survey. Rev Saúde Publica 1993;27(2):87-94.

3. Izquierdo MK, Hakkinen K, Antón A, Garrues M, Ibañez J, Ruesta M, et al. Maximal strength and power, endurance performance, and serum hormones in middle-aged and elderly men. Med Sci Sports Exerc 2001 33(9):1577-1587.

4. Sipila S, Koskinen SOA, Taafe DR, Takala TES, Cheng S, Rantanen T, et al. Determinants of lower-body muscle power in early postmenopausal women. J Am Geriatr Soc 2004;52(6):939-944.

5. American Collage of Sports Medicine. Exercise and Physical Activity for Older Adults. Med Sci Sports Exerc 1998;30(6):992-1008.

6. Gross MM, Stevenson PJ, Charette SL, Pyka G, Marcus R. Effect of muscle strength and movement speed on the biomechanics of rising from a chair in healthy elderly and young women. Gait Posture 1998;8(3):175-185.

7. American Collage of Sports Medicine, American Heart Association. Physical Activity and Public Health in Older Adults: Recommendation from the american college of sports medicine and the american heart association. Circulation 2007;116:1094-1105

8. Roelants MC, Delecluse C, Verschueren SM. Whole-bodyvibration training increases knee-extension strength and speed of movement in older women. J Am Geriatric Soc 2004; 52:901-908.

9. Vicent KR, Braith W, Feldman RA, Magyari MS, Rachel BC, Persin SA, et al. Resistance exercise and physical performance in adults aged 60 to 83. J Am Geriatr Soc 2002; 50(6):1100-1107.

10. Rikli R, Jones J. Senior fitness test manual. Champaign: Human Kinetics. 2001.

11. Rikli R, Jones J. Development and validation of a functional fitness test for community-residing older adults. J Aging Physical Activity 1999;7:129-161.

12. Bautmans I, Van HeesE, Lemper JC, Mets T. The feasibility of whole body vibration in institutionalised elderly persons and its influence on muscle performance, balance and mobility: a randomised controlled trial. BMC Geriatrics 2005; 5:17.

13. Bruyere O, Wuidart MA, Di Palma E, Gourlay M, Ethgen $\mathrm{O}$, Richy F, et al. Controlled whole body vibration to decrease fall risk and improve health-related quality of life of nursing home residents. Arch Phys Med Rehabil 2005;86(2):303-307.

14. Cheung WH, Mok HW, Qin L, Sze PC, Lee KM, Leung KS. High-frequency whole-body vibration improves balancing ability in elderly women. Arch Phys Med Rehabil 2007;88(7):852-857.

15. Bischoff HA, Stähelin HA, Monsch AU, Iversen MD, Wey A, Dechend MV et al. Identifying a cut-off point for normal mobility: a comparison of the timed 'up and go' test in community-dwelling and institutionalised elderly women. Age Ageing 2003;32(3):315-20.

16. Kawanabe K, Kawashima A, Sashimoto I, Takeda T., Sato, Yoshihiro, Iwamamoto J. Effect of whole-body vi- 
bration exercise and muscle strengthening, balance, and walking exercises on walking ability in the elderly. J Med 2007;56(1):28-33.

17. Shmway-Cook A, Brauer S, Woolacott M. Predicting the probability for falls in community-dwelling older adults using the timed up \& go test. Phys Ther 2000; 80(9): 896-903.

18. Bogaerts A, Verschueren, S, Delecluse C, Claessens AL, Boonen S. Effects of whole body vibration training on postural control in older individuals: a 1 year randomized controlled trial. Gait Posture 2007;26(2):309-316.

19. Fagnani F, Giombini A, Di Cesare A, Pigozzi F, Di Salvo V. The effects of whole-body vibration programo $n$ muscle performance and flexibility in female athletes. Am J Med Rehabil 2006; 85(12):956-962.

20. Issurin VB, Liebermann DG, Tenebaum G. Effect of vibratory stimulation training on maximal force and flexibility. J Sports Sci 1994;12(6):561-566.

21. Russo CR, Lauretani F, Bandinelli S, Bartali B, Cavazzini C, Guralnik JM, Ferrucci L. High-frequency vibration training increases muscle Power in postmenopausal women. Arch Phys Med Rehabil 2003; 84(11):1854-1857.

22. Verschueren SM, Roelants M, Delecluse C, Swinnen S, Vanderschueren D, Boonen S. Effect of 6-month whole body vibration training on hip density, muscle streng- th, and postural control in postmenopausal women: a randomized controlled pilot study. J Bone Miner Res 2004;19(3):352-359.

23. Delecluse C, Roelants M, Verschueren S. Strength increase after whole-body vibration compared with resistance training. Med Sci Sports Exerc 2003;35(6):1033-1041.

24. Ronnestad BR. Comparing the performance-enhancing effects of squats on a vibration platform with conventional squats in recreationally resistance-trained men. J Strength Cond Res 2004;18(4):839-845.

25. Shephard RJ. Aging, physical activity, and health. Champaign: Human Kinetics. 1997

\section{Endereço para correspondência \\ Carlos Ugrinowitsch}

Av. Prof. Mello Moraes, 65, Cidade Universitária, Butantã,

CEP: 05508-030 - São Paulo, SP

E-mail: ugrinowi@usp.br 